\title{
Prevalencia y factores asociados al consumo de tabaco, alcohol y drogas en una muestra poblacional de adultos mayores del Distrito Federal
}

\author{
Guilherme Luiz Guimaraes Borges, ${ }^{1}$ Miguel Ángel Mendoza Meléndez, ${ }^{2}$ Miguel Ángel López Brambila, ${ }^{2}$ \\ José Ángel García Pacheco,' Laura R. Velasco-Ángeles, ' María Angélica Beltrán Silva, ${ }^{2}$ \\ Pedro Eduardo Valdez Corchado, ${ }^{3}$ María Elena Medina-Mora, ${ }^{4}$ Rafael Camacho Solís ${ }^{2}$
}

Artículo original

\section{SUMMARY}

There are no studies in Mexico estimating the use of psychoactive substances in the elderly (+69 yrs) and there is a lack of information about the risk factors determining the burden of disease for this age group. The goal of this study is to present prevalences of use of alcohol, tobacco, illicit drugs and nonmedical prescription drugs in beneficiaries of a cash supplement program sponsored by the Institute for the Care of Elderly from Federal District (Instituto para la Atención del Adulto Mayor del Distrito Federal, IAAM-DF). Furthermore, significant sociodemographic factors associated with substance use are reported. From a random sample of the 2501 users registries of a cash supplement program of IAAM-DF, a total of 2098 agreed to participate and were interviewed in their homes. The response rate was of $83.9 \%$. We found that $63.5 \%$ of all participants ever used alcohol, $45.4 \%$ ever used tobacco and $3.5 \%$ ever used any drugs. Near one out of every two men and one out of every four women used alcohol during the previous 12 months, one out of every 10 older adults used tobacco and just one out of every 100 used any kind of drug in the last 12 months. Overall prevalence of substance use is higher in men, with the exception of medical drugs. Outstanding associated factors in substance use were sex and higher socioeconomic levels. This is the first study in the country on substance use in dwelling elderly, not living in institutions. Alcohol use, even risky drinking, is not uncommon, smoking tobacco habits do not disappear with age, and medical drug use can be of concern for its effects on other diseases. Therefore, it becomes necessary to establish definitions of risky use for this population and develop appropriate intervention programs for this population group.

Key words: Alcohol, tobacco, drugs, eldery, prevalence and associated factors.

\section{RESUMEN}

No existen investigaciones en el país sobre uso de sustancias psicoactivas en poblaciones de adultos mayores (+69 años) y se carece de información sobre los factores de riesgos que son determinantes para la carga de la enfermedad en este grupo. Este estudio tuvo como objetivo presentar las prevalencias de consumo de alcohol, tabaco, drogas ilícitas y drogas médicas usadas sin prescripción en los beneficiarios del programa de pensión alimentaria del Instituto para la Atención del Adulto Mayor del Distrito Federal (IAAM-DF). Adicionalmente, se reportaron factores sociodemográficos asociados al consumo de estas sustancias. De una muestra aleatoria del padrón de usuarios del programa del IAAM-DF de 2501 personas, un total de 2098 aceptaron participar y fueron entrevistadas en sus hogares, obteniéndose una tasa de respuesta de $83.9 \%$.

Se encontró que $65.3 \%$ de la población consumió alcohol, $45.4 \%$ tabaco y $3.5 \%$ alguna droga alguna vez en la vida. En los últimos 12 meses, casi uno de cada dos varones y una de cada cuatro mujeres consumió alcohol; uno de cada 10 adultos mayores consumió tabaco; y sólo uno de cada 100 consumió alguna droga. La prevalencia de consumo de sustancias es mayor en hombres, con excepción de las drogas médicas. Los factores asociados que destacaron fueron el sexo y los mejores niveles económicos.

Éste es el primer estudio realizado en el país sobre uso de sustancias en población de adultos mayores con domicilio fijo, no institucionalizado. El consumo de alcohol, incluso el consumo riesgoso, no es infrecuente; el hábito tabáquico no ha desaparecido con la edad, y el uso de drogas médicas puede ser un motivo de preocupación por su efecto en otras patologías. Se hace necesario establecer definiciones de uso riesgoso para esta población y desarrollar programas de intervención adecuados para este grupo.

Palabras clave: Alcohol, tabaco, drogas, adultos mayores, prevalencia y factores asociados.

\footnotetext{
Departamento de Modelos de Intervención. Instituto Nacional de Psiquiatría Ramón de la Fuente Muñiz, México.

2 Dirección Ejecutiva de Investigación y Evaluación. Instituto para la Atención y Prevención de las Adicciones en la Ciudad de México (IAPA), México.

3 Coordinación de Geriatría. Instituto para la Atención de los Adultos Mayores en el Distrito Federal (IAAM-DF), México.

Dirección General. Instituto Nacional de Psiquiatría Ramón de la Fuente Muñiz، México.
}

Correspondencia: Dr. Guilherme Borges. Dirección de Investigaciones Epidemiológicas y Psicosociales. Instituto Nacional de Psiquiatría Ramón de la Fuente Muñiz. Calz. México-Xochimilco 101, San Lorenzo Huipulco, Tlalpan, 14370, México D.F. E-mail: guibor@imp.edu.mx

Recibido primera versión: 3 de septiembre de 2013. Segunda versión: 29 de octubre de 2013. Aceptado: 13 de noviembre de 2013. 


\section{INTRODUCCIÓN}

Pese a que desde hace más de 30 años en el país, y en particular en la Ciudad de México, ${ }^{1}$ se han realizado muchas investigaciones para conocer el consumo de alcohol y de otras sustancias como tabaco, drogas ilícitas y uso de medicamentos sin prescripción médica tanto en la población adulta como en la de los adolescentes, ninguna de ellas ha incluido en su muestra poblacional a personas mayores de 69 años, es decir, a los adultos mayores. Ni siquiera aquellas investigaciones que se han realizado para conocer las repercusiones que ejercen estas sustancias sobre la salud han considerado a este segmento poblacional. Estas investigaciones en su mayoría, incluida la más reciente Encuesta Nacional de Adicciones, ${ }^{2-4}$ tienen como límite superior de edad los 65 años de edad. Sólo recientemente, algunas encuestas generales de salud en el país ${ }^{5,6}$ o en la Ciudad de México ${ }^{7}$ han incluido a la población de la tercera edad en su marco muestral, reportando prevalencias muy generales sobre consumo de alcohol o tabaco. Hasta ahora en ninguna de las investigaciones realizadas en adultos mayores se han reportado o considerado las prevalencias de uso de drogas, ya sean éstas ilícitas o médicas sin prescripción.

Es necesario, por lo tanto, hacer un diagnóstico de la situación del consumo de sustancias en personas de la tercera edad. Sobre todo si se considera que gran parte del perfil epidemiológico y de la carga de enfermedad en nuestro país está relacionado con el consumo de alcohol, tabaco y otras sustancias, así como con los problemas de salud derivados de ellas que impactan justamente en edades avanzadas (cáncer, enfermedades circulatorias, respiratorias o neurodegenerativas, etc.). ${ }^{8}$

De acuerdo con el INEGI, en el año 2010 había 496567 personas adultas en el Distrito Federal con edades iguales o mayores a los 69 años de edad. ${ }^{9}$ En octubre de ese mismo año, el IAAM-DF estimó que un total de 458775 personas eran beneficiarias del programa de pensión alimentaria. En esta estimación se incluyó a la población de adultos mayores de todos los estratos sociales, es decir, alrededor de $92.4 \%$ de toda la población de este grupo etario. ${ }^{10}$ Según proyecciones del CONAPO, para el año 2012 había 526230 personas adultas de la población del Distrito Federal con edades iguales o mayores a los 69 años de edad. ${ }^{11}$ De acuerdo con estimaciones del IAAM-DF, para julio de 2012 un total de 480000 personas habían sido beneficiarias del programa de pensión alimentaria. En estas cifras fue incluida la población de adultos mayores de todos los estratos sociales, es decir, alrededor de $91.2 \%$ de toda la población de este grupo etario. ${ }^{12} \mathrm{El}$ proceso de envejecimiento por el que atraviesa la población nacional, ${ }^{13,14}$ especialmente aquella que se concentra en grandes centros urbanos como la Ciudad de México, ${ }^{15}$ representará en un tiempo no muy lejano nuevos retos para la atención de salud en esta población. Lo anterior muestra la necesidad de conocer en detalle los factores de riesgo que son determinantes para la carga de enfermedad con respec- to al consumo de tabaco, de alcohol y, en menor medida, de sustancias psicoactivas en esta población.

Así, este estudio tiene como objetivo reportar las prevalencias de consumo de alcohol, tabaco, drogas ilícitas y drogas médicas usadas sin prescripción en adultos mayores del Distrito Federal (personas de 69 y más) beneficiarios del programa de pensión alimentaria del Instituto para la Atención del Adulto Mayor del Distrito Federal (IAAM-DF). Junto a las prevalencias de uso de sustancias, se reportarán factores sociodemográficos asociados a su consumo.

\section{MÉTODO}

La población blanco de este estudio fueron los adultos mayores que hasta julio de 2012 habían estado viviendo en el Distrito Federal y que eran beneficiarios del programa de pensión alimentaria para adultos mayores que estaba siendo implementado por el IAAM-DF.

La inclusión en este programa no requirió más que la suma voluntaria a éste y toda persona que pudo comprobar haber residido en el Distrito Federal desde hace tres años o más y que ya había cumplido los 69 años de edad era incorporada al programa. El IAAM-DF lleva los registros de los que son beneficiaros de este programa y en su sitio de Internet se encuentra publicado su padrón de derechohabientes que, gracias a la Ley de Transparencia y Acceso a la Información Pública del Distrito Federal, está abierta al público y es actualizada mensualmente. El presente estudio, con la autorización y otras facilidades otorgadas por el IAAM-DF, hizo uso de dicho padrón.

Todas las personas registradas al mes de julio del año 2012 conformaron el marco muestral de este estudio. El Instituto para la Atención y Prevención de las Adicciones (IAPA) de la Ciudad de México realizó un muestreo aleatorio entre los beneficiarios del programa de pensión alimentaria para adultos mayores. Los estratos de ese muestreo fueron: delegación de residencia, grupo de edad y género. La tasa de norespuesta estimada fue de $20 \%$ y las prevalencias más bajas estimadas fueron cercanas a $0.08 \%$.

Finalmente, de los 480000 beneficiarios se seleccionó una muestra aleatoria de 2501 adultos mayores de ambos sexos, residentes de alguna de las 16 delegaciones de la Ciudad de México que formaban parte del padrón del IAAM-DF. A todos los adultos mayores seleccionados se les aplicaron dos instrumentos, para lo cual se capacitó a educadores comunitarios que visitaron a los adultos mayores (sólo uno por hogar) y llevaron a cabo la aplicación de los instrumentos. Los educadores comunitarios son personas que se encuentran en cada una de las jurisdicciones sanitarias de las delegaciones y cuya función es la de realizar visitas domiciliarias regulares y entrevistar periódicamente a los adultos mayores beneficiarios de alguno de estos programas. Para esta investigación, el personal del IAPA capacitó en agosto de 2012 a los educa- 
dores comunitarios de cada una de las 16 delegaciones del Distrito Federal en el manejo de: 1. la Encuesta sobre Consumo de Sustancias Psicoactivas en Adultos Mayores en la Ciudad de México, y 2) la aplicación de la Cédula de Identificación de Riesgos en personas Adultas Mayores. Puesto que los reactivos del cuestionario de la presente encuesta pueden considerarse como una versión resumida de la Encuesta Nacional de Adicciones 2011 (ENA 2011), sus resultados pueden ser comparables con los de aquélla.

\section{Aspectos éticos}

Se entrevistó a todos los individuos resultantes del muestreo aleatorio simple explicado anteriormente, pero sólo después de que leyeron (o les fue leída, en caso necesario) una carta de consentimiento informado. Sólo quienes aceptaron participar fueron considerados para este estudio. En todo momento se hizo énfasis en el carácter voluntario de su participación y en el manejo confidencial de la información.

\section{Definición de variables principales}

Para los fines de este estudio, y de acuerdo con la ENA-2011, se definieron las siguientes variables:

\section{Alcohol}

- Abstemio: quien nunca consumió alcohol alguna vez en la vida.

- Bebedor: quien alguna vez en la vida consumió alcohol.

- Bebedor actual: quien ha consumido alcohol en los últimos 12 meses.

- Consumo riesgoso: consumo de cinco o más copas en hombres, cuatro o más en mujeres, al menos una vez al año durante el último año.

- Abuso o dependencia al alcohol: definidos conforme a los criterios de clasificación del DSM-IV-TR Manual diagnóstico y estadístico de los trastornos mentales (Diagnostic and Statistical Manual of Mental Disorders). ${ }^{16}$

\section{Tabaco}

- Consumidor de tabaco: quien alguna vez en la vida consumió tabaco.

- Consumidor de 100 o más cigarros: quien alguna vez en la vida consumió más de 100 cigarros, es decir, cinco cajetillas.

- Fumador activo: quien fumó por lo menos una vez al año en los últimos 12 meses.

\section{Drogas}

- Drogas médicas sin prescripción: incluye el consumo de opiáceos, tranquilizantes, sedantes y barbitúricos, anfetaminas y estimulantes sin receta o en forma diferente a lo indicado por un médico (en mayor cantidad, por más tiempo o cualquier situación diferente a la indicada).
- Drogas ilícitas: incluye el consumo de marihuana, hashish, cocaína, crack, alucinógenos, inhalables, heroína, opio, estimulantes tipo anfetamínico, otras drogas.

- Drogas: considérese aquí el consumo de drogas médicas o drogas ilícitas.

Con respecto a la edad, para fines de este trabajo se dividió a la población de adultos mayores en tercera edad (6979 años) y cuarta edad (80-106 años).

\section{Análisis de datos}

Se reportan las prevalencias de consumo de sustancias (alcohol, tabaco y drogas) de alguna vez en la vida y en los últimos 12 meses para una serie de variables sociodemográficas; se incluyen pruebas de Chi-cuadrada $\left(\chi^{2}\right)$ y la probabilidad asociada a ésta. Se incluyen también los resultados de modelos multivariados para medir la asociación entre los principales factores sociodemográficos y las prevalencias de uso de sustancias, ${ }_{17}^{17}$ por medio de la razón de momios (odds ratio).

\section{RESULTADOS}

En la muestra de 2501 personas del listado de 480000 beneficiarios del programa de pensión alimentaria del Distrito Federal no se encontraron diferencias por grupos por edad y sexo entre esta muestra y la población de beneficiarios. Un total de 2098 aceptaron participar y fueron entrevistados. La tasa de respuesta fue de $83.9 \%$ y varió desde $72.3 \%$ en la delegación Miguel Hidalgo hasta $93.5 \%$ en la delegación Xochimilco. No se encontraron diferencias estadísticamente significativas por grupos de edad ni por sexo entre los 2098 participantes y la población blanco de adultos mayores (datos disponibles por el autor).

La distribución de los participantes, por sexo y variables demográficas, se encuentra en el cuadro 1. Casi dos terceras partes de la muestra la conforman mujeres, mientras que del total de la muestra, $66.2 \%$ estuvo entre 69-79 años. Un poco menos de la mitad estaba casado o vivía en unión libre, y sólo $41.3 \%$ tenía estudios de primaria incompleta o menos. Como se puede observar, ésta es una población de escasos recursos económicos, pues poco más de 70\% recibe cuatro salarios mínimos o menos, aunque $80.9 \%$ tiene algún tipo de seguro médico. Aunque $79.2 \%$ reporta no necesitar ni tener un cuidador, sólo $23.7 \%$ reporta una salud "excelente" y $60.2 \%$ tiene por lo menos una enfermedad diagnosticada por algún médico.

El cuadro 2 presenta las prevalencias de uso de sustancias alguna vez en la vida y en los últimos 12 meses, por sexo. Como se puede observar, con excepción del uso de drogas médicas alguna vez en la vida y uso de drogas los últimos 12 meses, en todas las otras prevalencias los hombres consumen más alcohol, tabaco y drogas que las mujeres. El 65.3\% de la población consumió alcohol, $45.4 \%$ consumió tabaco y 3.5\% consumió alguna droga alguna vez en la vida. El consumo actual de estas sustancias es mucho menos frecuente, pero aun 
Cuadro 1. Descriptivos sociodemográficos y de salud de la muestra por sexo, encuesta del Uso de Sustancias en Adultos Mayores del Distrito Federal, USAMDF-2012 ( $\mathrm{n}=2098)$

\begin{tabular}{|c|c|c|c|c|}
\hline & $\begin{array}{c}\text { Masculino } \\
(\mathrm{n}=799)\end{array}$ & $\begin{array}{l}\text { Femenino } \\
(n=1299)\end{array}$ & $\begin{array}{c}\text { Total } \\
(n=2098)\end{array}$ & \\
\hline Sexo & 38.10 & 61.90 & 100.00 & \\
\hline $\begin{array}{l}\text { Grupo de edad } \\
\text { - Tercera edad } \\
\text { - Cuarta edad }\end{array}$ & $\begin{array}{l}68.50 \\
31.50\end{array}$ & $\begin{array}{l}64.80 \\
35.20\end{array}$ & $\begin{array}{l}66.20 \\
33.80\end{array}$ & $\chi^{2}(1)=2.93 \operatorname{Pr}=0.087$ \\
\hline $\begin{array}{l}\text { Estado civil } \\
\text { - Otro } \\
\text { - Casado/unido }\end{array}$ & $\begin{array}{l}32.00 \\
68.00\end{array}$ & $\begin{array}{l}70.90 \\
29.10\end{array}$ & $\begin{array}{l}56.10 \\
43.90\end{array}$ & $\chi^{2}(1)=302.63 \operatorname{Pr}=0.000$ \\
\hline $\begin{array}{l}\text { Grado escolar } \\
\text { - Primaria incompleta o menos } \\
\text { - Primaria o secundaria (completa o incompleta) } \\
\text { - Preparatoria o más }\end{array}$ & $\begin{array}{l}35.50 \\
37.60 \\
26.90\end{array}$ & $\begin{array}{l}45.00 \\
39.30 \\
15.70\end{array}$ & $\begin{array}{l}41.30 \\
38.70 \\
20.00\end{array}$ & $\chi^{2}(2)=40.20 \operatorname{Pr}=0.000$ \\
\hline $\begin{array}{l}\text { Ingresos } \\
\text { - Igual o menos un SM } \\
\text { - } 2 \text { SM } \\
\text { - De } 2 \text { a } 4 \text { SM } \\
\text { - Más de } 4 \text { SM }\end{array}$ & $\begin{array}{l}36.20 \\
30.20 \\
19.00 \\
14.50\end{array}$ & $\begin{array}{r}48.90 \\
26.50 \\
16.60 \\
8.00\end{array}$ & $\begin{array}{l}44.00 \\
27.90 \\
17.50 \\
10.50\end{array}$ & $\chi^{2}(3)=40.87 \operatorname{Pr}=0.000$ \\
\hline $\begin{array}{l}\text { Seguro } \\
\cdot \text { No } \\
\cdot \text { Sí }\end{array}$ & $\begin{array}{l}13.40 \\
86.60\end{array}$ & $\begin{array}{l}22.70 \\
77.30\end{array}$ & $\begin{array}{l}19.10 \\
80.90\end{array}$ & $\chi^{2}(1)=26.91 \operatorname{Pr}=0.000$ \\
\hline $\begin{array}{l}\text { Cuidador } \\
\text { - No necesita y no tiene } \\
\text { - No necesita pero tiene } \\
\text { - Necesita pero no tiene } \\
\text { - Necesita y tiene }\end{array}$ & $\begin{array}{r}82.60 \\
5.50 \\
2.40 \\
9.60\end{array}$ & $\begin{array}{r}77.10 \\
5.50 \\
3.70 \\
13.70\end{array}$ & $\begin{array}{r}79.20 \\
5.50 \\
3.20 \\
12.10\end{array}$ & $\chi^{2}(3)=11.20 \operatorname{Pr}=0.011$ \\
\hline $\begin{array}{l}\text { Percepción de la salud } \\
\text { - Excelente } \\
\text { - Muy buena } \\
\text { - Buena }\end{array}$ & $\begin{array}{l}26.50 \\
63.30 \\
10.30\end{array}$ & $\begin{array}{l}22.00 \\
62.40 \\
15.70\end{array}$ & $\begin{array}{l}23.70 \\
62.70 \\
13.60\end{array}$ & $\chi^{2}(2)=14.10 \operatorname{Pr}=0.001$ \\
\hline $\begin{array}{l}\text { Al menos una enfermedad diagnosticada } \\
\text { - Ninguna enfermedad diagnosticada } \\
\text { - Al menos una enfermedad diagnosticada }\end{array}$ & $\begin{array}{l}44.90 \\
55.10\end{array}$ & $\begin{array}{l}36.60 \\
63.40\end{array}$ & $\begin{array}{l}39.80 \\
60.20\end{array}$ & $\chi^{2}(1)=14.45 \operatorname{Pr}=0.000$ \\
\hline $\begin{array}{l}\text { Región } \\
\text { - Norte } \\
\text { - Oriente } \\
\text { - Poniente } \\
\text { - Sur }\end{array}$ & $\begin{array}{l}29.16 \\
38.17 \\
18.27 \\
14.39\end{array}$ & $\begin{array}{l}28.71 \\
36.95 \\
21.32 \\
13.01\end{array}$ & $\begin{array}{l}28.88 \\
37.42 \\
20.16 \\
13.54\end{array}$ & $\chi^{2}(3)=3.21 \operatorname{Pr}=0.360$ \\
\hline
\end{tabular}

Las regiones están catalogadas por: Norte: Azcapotzalco, Cuauhtémoc y Gustavo A. Madero; Oriente: Coyoacán, Izłacalco, Izłapalapa; Poniente: Álvaro Obregón, Benito Juárez, Cuajimalpa, Magdalena Contreras, Miguel Hidalgo y Sur: Milpa Alta, Tláhuac, Tlalpan y Xochimilco.

*** Trastornos mentales y del comportamiento, incluye trastorno bipolar, esquizofrenia, depresión y Alzheimer.

Enfermedades crónico-degenerativas, incluye diabetes mellitus, síndrome metabólico, disfunción eréctil, osteoporosis, esclerosis lateral amiotrófica, úlcera duodenal, ceguera, cataratas, glaucoma, cáncer de mama, cáncer de próstata, cáncer gástrico y cáncer de vejiga.

Enfermedades infecciosas/respiratorias, incluye neumonía, influenza, resfriado común, otitis media y virus papiloma $\mathrm{H}$.

$\mathrm{SM}=$ Salario mínimo

así significativo. Casi uno de cada dos varones y una de cada cuatro mujeres consumieron alcohol en los 12 meses previos; uno de cada 10 adultos mayores consumió tabaco y sólo uno de cada 100 consumió alguna droga. Aun cuando 8.7\% de la población (15.8\% de los hombres y $4.4 \%$ de las mujeres) reportó consumo riesgoso de alcohol en los últimos 12 meses, la prevalencia de cuadros clínicos de abuso/dependencia al alcohol sólo fue reportada por el 1.2\% en los últimos 12 meses.

El cuadro 3 presenta las principales variables asociadas con el consumo de alcohol en los últimos 12 meses, periodo en el cual se ha concentrado el análisis por motivos de espacio y para mostrar similitudes con el consumo de alcohol alguna vez en la vida. La única variable que estuvo consistentemente asociada con incrementos en la razón de momios (RM) para el consumo, el consumo riesgoso y el abuso/dependencia al alcohol fue el sexo masculino. Las personas con más de cuatro salarios mínimos reportaron incrementos en la RM para el consumo y el consumo riesgoso en los últimos 12 meses y aquellas personas que reportaron la presencia de una o más enfermedades diagnosticadas reportaron incre- 
Cuadro 2. Descriptivos de consumo de sustancias en adultos mayores del Distrito Federal por sexo, USAMDF-2012

\begin{tabular}{|c|c|c|c|c|}
\hline & $\begin{array}{c}\text { Masculino } \\
(n=799)\end{array}$ & $\begin{array}{l}\text { Femenino } \\
(n=1299)\end{array}$ & $\begin{array}{c}\text { Total } \\
(\mathrm{n}=2098)\end{array}$ & \\
\hline Consumo de alcohol alguna vez & & & & $\chi^{2}(1)=275.00 \operatorname{Pr}=0.000$ \\
\hline - No & 12.8 & 48.3 & 34.7 & \\
\hline • Sí & 87.2 & 51.7 & 65.3 & \\
\hline Consumo de alcohol en los últimos 12 meses & & & & $\chi^{2}(1)=83.77 \operatorname{Pr}=0.000$ \\
\hline$\cdot$ No & 55.8 & 75.1 & 67.7 & \\
\hline - Sí & 44.2 & 24.9 & 32.3 & \\
\hline Consumo riesgoso alguna vez en la vida & & & & $\chi^{2}(1)=165.73 \operatorname{Pr}=0.000$ \\
\hline - No & 56.9 & 82.7 & 72.9 & \\
\hline - Sí & 43.1 & 17.3 & 27.1 & \\
\hline Consumo riesgoso anual & & & & $\chi^{2}(1)=148.92 \operatorname{Pr}=0.000$ \\
\hline - No & 71.8 & 91.8 & 84.2 & \\
\hline - Sí & 28.2 & 8.2 & 15.8 & \\
\hline Consumo riesgoso mensual & & & & $\chi^{2}(1)=80.49 \operatorname{Pr}=0.000$ \\
\hline - No & 84.2 & 95.6 & 91.3 & \\
\hline - Sí & 15.8 & 4.4 & 8.7 & \\
\hline Abuso o dependencia de alcohol alguna vez en la vida & & & & $\chi^{2}(1)=193.64 \operatorname{Pr}=0.000$ \\
\hline - No & 80.0 & 97.8 & 91.0 & \\
\hline - Sí & 20.0 & 2.2 & 9.0 & \\
\hline Abuso o dependencia de alcohol 12 meses & & & & $\chi^{2}(1)=28.33 \operatorname{Pr}=0.000$ \\
\hline - No & 97.1 & 99.8 & 98.8 & \\
\hline - Sí & 2.9 & 0.2 & 1.2 & \\
\hline Consumo de tabaco alguna vez en la vida & & & & $\chi^{2}(1)=337.53 \operatorname{Pr}=0.000$ \\
\hline - No & 29.2 & 70.3 & 54.6 & \\
\hline - Sí & 70.8 & 29.7 & 45.4 & \\
\hline Consumo de 100 cigarros o más en la vida & & & & $\chi^{2}(1)=259.44 \operatorname{Pr}=0.000$ \\
\hline - No & 50.6 & 83.4 & 70.9 & \\
\hline - Sí & 49.4 & 16.6 & 29.1 & \\
\hline Consumo de tabaco en los últimos 12 meses & & & & $\chi^{2}(1)=52.48 \operatorname{Pr}=0.000$ \\
\hline - No & 84.6 & 94.1 & 90.5 & \\
\hline - Sí & 15.4 & 5.9 & 9.5 & \\
\hline Cualquier droga alguna vez en la vida & & & & $\chi^{2}(1)=6.95 \operatorname{Pr}=0.008$ \\
\hline - No & 95.1 & 97.3 & 96.5 & \\
\hline - Sí & 4.9 & 2.7 & 3.5 & \\
\hline Cualquier droga médica alguna vez en la vida & & & & $\chi^{2}(1)=3.06 \operatorname{Pr}=0.080$ \\
\hline - No & 99.4 & 98.5 & 98.9 & \\
\hline • Sí & 0.6 & 1.5 & 1.1 & \\
\hline Cualquier droga ilegal alguna vez en la vida & & & & $\chi^{2}(1)=19.44 \operatorname{Pr}=0.000$ \\
\hline - No & 95.7 & 98.8 & 97.6 & \\
\hline • Sí & 4.3 & 1.2 & 2.4 & \\
\hline Cualquier droga en los últimos 12 meses & & & & $\chi^{2}(1)=0.03 \operatorname{Pr}=0.867$ \\
\hline - No & 99.0 & 98.9 & 99.0 & \\
\hline - Sí & 1.0 & 1.1 & 1.0 & \\
\hline
\end{tabular}

mentos en la RM para el consumo y el consumo riesgoso en los últimos 12 meses. Otras variables asociadas únicamente con el consumo en los últimos 12 meses fueron las siguientes: aquellas personas con preparatoria o más, los entrevistados en las regiones poniente y sur de la Ciudad, y aquellos que reportaron una mala salud tuvieron RM menores que aquellos con una salud excelente. Solamente el sexo masculino estuvo asociado en el abuso o dependencia al alcohol en los últimos 12 meses, luego de haber controlado por las variables de salud y sociodemográficas.

Para el consumo de tabaco (cuadro 4) las únicas variables que estuvieron consistentemente asociadas con incrementos en la RM fueron el sexo masculino y niveles más elevados de estudios (preparatoria o más). Aquellas personas que reportaron la presencia de una o más enfermedades diagnósticas reportaron incrementos en la RM para consumo de tabaco alguna vez y para el consumo de 100 o más cigarrillos en la vida. Aquellas personas con dos salarios mínimos reportaron menores RM para el consumo de tabaco en los últimos 12 meses.

El cuadro 5 muestra los factores asociados con el consumo de cualquier droga alguna vez en la vida y en los últimos 12 meses. La única variable que estuvo consistentemente asociada con incrementos en la RM para el consumo fue el sexo masculino. Aquellas personas que ganaban de 2-4 salarios mínimos reportaron, a la vez, incrementos en la 
Guimaraes Borges et al.

Cuadro 3. Análisis multivariado de variables sociodemográficas asociadas al consumo de alcohol en los últimos 12 meses, USAMDF-2012

\begin{tabular}{|c|c|c|c|c|c|c|}
\hline & \multicolumn{2}{|c|}{ Consumo } & \multicolumn{2}{|c|}{ Consumo riesgoso } & \multicolumn{2}{|c|}{ Abuso o dependencia } \\
\hline & RM & IC95 & RM & IC95 & RM & IC95 \\
\hline \multicolumn{7}{|l|}{ Sexo } \\
\hline - Femenino & 1.00 & & 1.00 & - & 1.00 & - \\
\hline - Masculino & 2.18 & $(1.72-2.76)$ & 4.70 & $(3.43-6.45)$ & 11.31 & $(3.06-41.72)$ \\
\hline \multicolumn{7}{|l|}{ Grupos de edad } \\
\hline - Tercera edad & 1.00 & - & 1.00 & - & 1.00 & - \\
\hline - Cuarta edad & 0.88 & $(0.69-1.12)$ & 1.03 & $(0.76-1.41)$ & 0.61 & $(0.20-1.82)$ \\
\hline \multicolumn{7}{|l|}{ Estado civil } \\
\hline - Unido & 1.00 & - & 1.00 & - & 1.00 & - \\
\hline - No unido & 0.99 & $(0.78-1.26)$ & 1.06 & $(0.78-1.45)$ & 0.89 & $(0.33-2.38)$ \\
\hline \multicolumn{7}{|l|}{ Grado escolar } \\
\hline - Primaria incompleta o menos & 1.00 & - & 1.00 & - & 1.00 & - \\
\hline - Primaria o secundaria (completa o incompleta) & 1.19 & $(0.93-1.52)$ & 0.99 & $(0.72-1.35)$ & 1.69 & $(0.62-4.66)$ \\
\hline - Preparatoria o más & 1.56 & $(1.15-2.13)$ & 0.71 & (0.47-1.09) & 0.87 & $(0.20-3.83)$ \\
\hline \multicolumn{7}{|l|}{ Ingresos } \\
\hline - Igual o menos de un SM & 1.00 & - & 1.00 & - & 1.00 & - \\
\hline$\cdot 2$ SM & 1.41 & $(1.09-1.84)$ & 1.26 & $(0.89-1.77)$ & 0.52 & $(0.17-1.6)$ \\
\hline - de 2 a 4 SM & 1.72 & $(1.27-2.33)$ & 1.41 & $(0.94-2.12)$ & 0.70 & $(0.20-2.43)$ \\
\hline - Más de 4 SM & 2.24 & (1.54-3.24) & 1.64 & $(1.01-2.65)$ & 0.50 & $(0.10-2.65)$ \\
\hline \multicolumn{7}{|l|}{ Seguro } \\
\hline - No & 1.00 & - & 1.00 & - & 1.00 & - \\
\hline • Sí & 1.33 & (0.99-1.78) & 1.04 & $(0.71-1.53)$ & 4.14 & $(0.54-31.93)$ \\
\hline \multicolumn{7}{|l|}{ Cuidador } \\
\hline - No necesita y no tiene & 1.00 & - & 1.00 & - & 1.00 & - \\
\hline - No necesita pero tiene & 1.21 & $(0.77-1.91)$ & 5.69 & $(3.56-9.11)$ & 1.66 & $(0.35-7.82)$ \\
\hline - Necesita pero no tiene & 0.84 & (0.42-1.65) & 0.91 & $(0.36-2.26)$ & 1.49 & $(0.17-13.14)$ \\
\hline - Necesita y tiene & 0.72 & (0.49-1.05) & 0.72 & $(0.42-1.23)$ & 0.97 & $(0.19-4.89)$ \\
\hline \multicolumn{7}{|l|}{ Percepción de la salud } \\
\hline - Excelente & 1.00 & - & 1.00 & - & 1.00 & - \\
\hline - Buena & 1.00 & $(0.78-1.28)$ & 1.30 & (0.93-1.83) & 1.19 & $(0.37-3.8)$ \\
\hline - Mala & 0.64 & (0.42-0.97) & 0.87 & $(0.50-1.52)$ & 1.99 & (0.44-9.08) \\
\hline \multicolumn{7}{|l|}{ Cualquier enfermedad } \\
\hline - Ninguna enfermedad & 1.00 & - & 1.00 & - & 1.00 & - \\
\hline - Al menos una enfermedad* & 1.31 & $(1.05-1.64)$ & 1.31 & $(0.98-1.76)$ & 1.54 & (0.59-4.07) \\
\hline \multicolumn{7}{|l|}{ Región } \\
\hline - Norte & 1.00 & - & 1.00 & - & 1.00 & - \\
\hline - Oriente & 0.97 & $(0.74-1.26)$ & 0.90 & (0.64-1.28) & 1.01 & $(0.34-2.96)$ \\
\hline - Poniente & 1.56 & $(1.15-2.11)$ & 1.27 & $(0.86-1.88)$ & 0.29 & (0.03-2.47) \\
\hline - Sur & 1.72 & $(1.22-2.42)$ & 0.94 & $(0.60-1.48)$ & 1.67 & $(0.49-5.73)$ \\
\hline
\end{tabular}

El grupo de tercera edad incluye a las personas con una edad de 68-74 años y el grupo de la cuarta edad son aquellos con una edad igual o mayor de 75 años. Las regiones están catalogadas por: Norte: Azcapotzalco, Cuauhtémoc y Gustavo A. Madero; Oriente: Coyoacán, Iztacalco, Iztapalapa; Poniente: Álvaro Obregón, Benito Juárez, Cuajimalpa, Magdalena Contreras, Miguel Hidalgo y Sur: Milpa Alta, Tláhuac, Tlalpan y Xochimilco.

* Trastornos mentales y del comportamiento, incluye trastorno bipolar, esquizofrenia, depresión y Alzheimer.

Enfermedades crónico-degenerativas, incluye diabetes melliłus, síndrome metabólico, disfunción eréctil, osteoporosis, esclerosis lateral amiotrófica, úlcera duodenal, ceguera, cataratas, glaucoma, cáncer de mama, cáncer de próstata, cáncer gástrico y cáncer de vejiga.

Enfermedades infecciosas/respiratorias, incluye neumonía, influenza, resfriado común, otitis media y virus papiloma $\mathrm{H}$.

$\mathrm{RM}=$ Razón de momios; $\mathrm{SM}=$ Salario mínimo.

RM para el consumo de alguna vez, y aquellas "sin unión" tuvieron menor RM para el consumo de alguna vez en la vida (RM no significativa).

\section{DISCUSIÓN}

En este trabajo se ha encontrado que $65.3 \%$ de la población consumió alcohol, $45.4 \%$ tabaco y $3.5 \%$ alguna droga alguna vez en la vida. Casi uno de cada dos varones y una de cada cuatro mujeres consumieron alcohol en los 12 meses previos; uno de cada 10 adultos mayores consumió tabaco y sólo uno de cada 100 consumió alguna droga. Este consumo no parece variar entre los grupos de la tercera y la cuarta edad. Por lo general, la prevalencia de consumo de sustancias es mayor en hombres, con excepción del de drogas médicas, que es mayor entre las mujeres. Pocos factores, además del sexo, se asociaron con estas prevalencias. Finalmente, se encontró una ligera tendencia en personas de mejores niveles socioeconómicos a consumir más sustancias. 
Cuadro 4. Análisis multivariado de variables sociodemográficas asociadas al consumo de tabaco alguna vez en la vida y en los últimos 12 meses, USAMDF-2012

\begin{tabular}{|c|c|c|c|c|c|c|}
\hline & \multicolumn{2}{|c|}{$\begin{array}{c}\text { Consumo de tabaco } \\
\text { alguna vez }\end{array}$} & \multicolumn{2}{|c|}{$\begin{array}{c}100 \text { cigarrillos } \\
\text { en la vida }\end{array}$} & \multicolumn{2}{|c|}{$\begin{array}{c}\text { Fumar en } \\
\text { los últimos } 12 \text { meses }\end{array}$} \\
\hline & RM & IC95 & $R M$ & IC95 & RM & IC95 \\
\hline $\begin{array}{l}\text { Sexo } \\
\text { - Femenino } \\
\text { - Masculino }\end{array}$ & $\begin{array}{l}1.00 \\
5.81\end{array}$ & $\begin{array}{c}- \\
(4.58-7.36)\end{array}$ & $\begin{array}{l}1.00 \\
5.04\end{array}$ & (3.93-6.45) & $\begin{array}{l}1.00 \\
2.88\end{array}$ & $(2.00-4.15)$ \\
\hline $\begin{array}{l}\text { Grupos de edad } \\
\text { - Tercera edad } \\
\text { - Cuarta edad }\end{array}$ & $\begin{array}{l}1.00 \\
0.87\end{array}$ & (0.69-1.10) & $\begin{array}{l}1.00 \\
0.88\end{array}$ & (0.69-1.14) & $\begin{array}{l}1.00 \\
0.43\end{array}$ & $(0.28-0.66)$ \\
\hline $\begin{array}{l}\text { Estado civil } \\
\text { - Unido } \\
\text { - No unido }\end{array}$ & $\begin{array}{l}1.00 \\
0.97\end{array}$ & $\begin{array}{c}- \\
(0.77-1.22)\end{array}$ & $\begin{array}{l}1.00 \\
1.04\end{array}$ & (0.81-1.33) & $\begin{array}{l}1.00 \\
0.74\end{array}$ & (0.52-1.07) \\
\hline $\begin{array}{l}\text { Grado escolar } \\
\text { - Menor o igual a primaria } \\
\text { - Primaria o secundaria (completa o incompleta) } \\
\text { - Preparatoria o más }\end{array}$ & $\begin{array}{l}1.00 \\
1.16 \\
1.46\end{array}$ & $\begin{array}{c}- \\
(0.92-1.47) \\
(1.06-2.00)\end{array}$ & $\begin{array}{l}1.00 \\
1.35 \\
1.41\end{array}$ & $\begin{array}{c}- \\
(1.05-1.75) \\
(1.01-1.96)\end{array}$ & $\begin{array}{l}1.00 \\
1.15 \\
1.75\end{array}$ & $\begin{array}{c}- \\
(0.78-1.70) \\
(1.10-2.79)\end{array}$ \\
\hline $\begin{array}{l}\text { Ingresos } \\
\text { - Igual o menos de un SM } \\
\text { - } 2 \text { SM } \\
\text { - de } 2 \text { a } 4 \text { SM } \\
\text { - Más de } 4 \text { SM }\end{array}$ & $\begin{array}{l}1.00 \\
1.28 \\
1.23 \\
1.27\end{array}$ & $\begin{array}{l}(0.99-1.65) \\
(0.91-1.68) \\
(0.87-1.87)\end{array}$ & $\begin{array}{l}1.00 \\
1.05 \\
1.05 \\
1.09\end{array}$ & $\begin{array}{l}- \\
(0.79-1.38) \\
(0.76-1.46) \\
(0.73-1.63)\end{array}$ & $\begin{array}{l}1.00 \\
0.64 \\
0.80 \\
0.84\end{array}$ & $\begin{array}{l}(0.42-0.98) \\
(0.50-1.28) \\
(0.48-1.47)\end{array}$ \\
\hline $\begin{array}{l}\text { Seguro } \\
\text { - No } \\
\text { - Sí }\end{array}$ & $\begin{array}{l}1.00 \\
1.04\end{array}$ & $\begin{array}{c}- \\
(0.79-1.36)\end{array}$ & $\begin{array}{l}1.00 \\
1.05\end{array}$ & (0.77-1.42) & $\begin{array}{l}1.00 \\
1.27\end{array}$ & (0.80-2.03) \\
\hline $\begin{array}{l}\text { Cuidador } \\
\text { - No necesita y no tiene } \\
\text { - No necesita pero tiene } \\
\text { - Necesita pero no tiene } \\
\text { - Necesita y tiene }\end{array}$ & $\begin{array}{l}1.00 \\
1.37 \\
1.41 \\
0.81\end{array}$ & $\begin{array}{c}- \\
(0.87-2.15) \\
(0.77-2.57) \\
(0.56-1.17)\end{array}$ & $\begin{array}{l}1.00 \\
1.16 \\
0.69 \\
0.78\end{array}$ & $\begin{array}{l}- \\
(0.72-1.87) \\
(0.34-1.40) \\
(0.52-1.17)\end{array}$ & $\begin{array}{l}1.00 \\
1.04 \\
0.57 \\
0.45\end{array}$ & $\begin{array}{c}- \\
(0.51-2.09) \\
(0.17-1.90) \\
(0.20-1.00)\end{array}$ \\
\hline $\begin{array}{l}\text { Percepción de la salud } \\
\text { - Excelente } \\
\text { - Buena } \\
\text { - Mala }\end{array}$ & $\begin{array}{l}1.00 \\
1.13 \\
1.28\end{array}$ & $\begin{array}{c}- \\
(0.88-1.46) \\
(0.88-1.88)\end{array}$ & $\begin{array}{l}1.00 \\
1.24 \\
1.23\end{array}$ & $\begin{array}{c}- \\
(0.94-1.62) \\
(0.81-1.87)\end{array}$ & $\begin{array}{l}1.00 \\
1.09 \\
1.02\end{array}$ & $\begin{array}{c}- \\
(0.74-1.60) \\
(0.54-1.93)\end{array}$ \\
\hline $\begin{array}{l}\text { Cualquier enfermedad } \\
\text { - Ninguna enfermedad } \\
\text { - Al menos una enfermedad* }\end{array}$ & $\begin{array}{l}1.00 \\
1.27\end{array}$ & $\begin{array}{c}- \\
\text { (1.02-1.59) }\end{array}$ & $\begin{array}{l}1.00 \\
1.54\end{array}$ & ${ }^{-} \cdot \overline{-}$ & $\begin{array}{l}1.00 \\
0.91\end{array}$ & (0.65-1.28) \\
\hline $\begin{array}{l}\text { Región } \\
\text { - Norte } \\
\text { - Oriente } \\
\text { - Poniente } \\
\text { - Sur }\end{array}$ & $\begin{array}{l}1.00 \\
1.03 \\
1.29 \\
1.13\end{array}$ & $\begin{array}{l}- \\
(0.79-1.33) \\
(0.95-1.75) \\
(0.80-1.59)\end{array}$ & $\begin{array}{l}1.00 \\
0.95 \\
1.11 \\
0.86\end{array}$ & $\begin{array}{c}- \\
(0.72-1.25) \\
(0.80-1.54) \\
(0.59-1.24)\end{array}$ & $\begin{array}{l}1.00 \\
1.09 \\
1.08 \\
1.07\end{array}$ & $\begin{array}{c}- \\
(0.73-1.64) \\
(0.67-1.74) \\
(0.63-1.82)\end{array}$ \\
\hline
\end{tabular}

El grupo de tercera edad incluye a las personas con una edad de $68-74$ años y el grupo de la cuarta edad son aquellos con una edad igual o mayor de 75 años.

Las regiones están catalogadas por: Norte: Azcapotzalco, Cuauhtémoc y Gustavo A. Madero; Oriente: Coyoacán, Iztacalco, Iztapalapa; Poniente: Álvaro Obregón, Benito Juárez, Cuajimalpa, Magdalena Contreras, Miguel Hidalgo y Sur: Milpa Alta, Tláhuac, Tlalpan y Xochimilco.

* Trastornos mentales y del comportamiento, incluye trastorno bipolar, esquizofrenia, depresión y Alzheimer.

Enfermedades crónico-degenerativas, incluye diabetes mellitus, síndrome metabólico, disfunción eréctil, osteoporosis, esclerosis lateral amiotrófica, úlcera duodenal, ceguera, cataratas, glaucoma, cáncer de mama, cáncer de próstata, cáncer gástrico y cáncer de vejiga.

Enfermedades infecciosas/respiratorias, incluye neumonía, influenza, resfriado común, otitis media y virus papiloma $\mathrm{H}$.

$\mathrm{RM}=$ Razón de momios; $\mathrm{SM}=$ Salario mínimo.

Las encuestas poblacionales en adultos mayores no son comunes en el país o en el escenario internacional. Muchas de las estimaciones previas que se han hecho sobre el uso de sustancias en adultos mayores se basan en poblaciones de tercera edad que consultaron a algún clínico ${ }^{18,19}$ o que están bajo cuidados especiales, en casas de reposo o albergues. ${ }^{20,21}$ En el país, con base en información del Estudio Nacional sobre Salud y Envejecimiento en México (ENASEM-2001), se estimó una prevalencia de consumo de alcohol y tabaco alguna vez en la vida en la población de 50 o más años de 61.4 y $42.7 \%,{ }^{22}$ respectivamente. ${ }^{23}$ Para aquellos que actualmente toman y fuman, estos autores reportaron en el mismo orden un consumo de 30.7 y $17.3 \% .{ }^{23}$ Otro estudio con la ENASEM (2001) estimó para adultos mayores de 65 años 
Cuadro 5. Análisis multivariado de variables sociodemográficas asociadas al consumo de drogas alguna vez en la vida y en los últimos 12 meses, USAMDF-2012

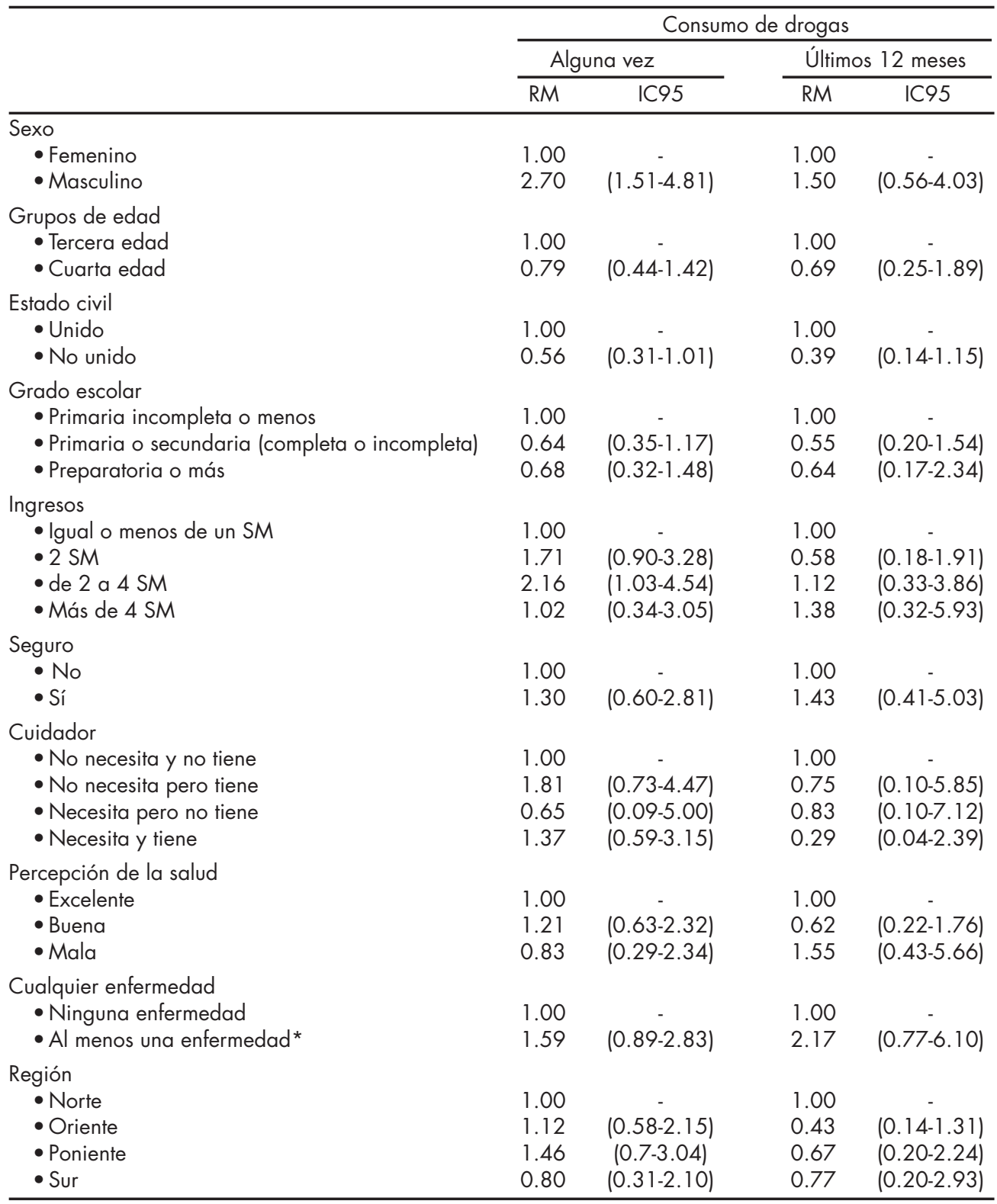

El grupo de tercera edad incluye a las personas con una edad de 68-74 años y el grupo de la cuarta edad son aquellos con una edad igual o mayor de 75 años.

Las regiones están catalogadas por: Norte: Azcapotzalco, Cuauhtémoc y Gustavo A. Madero; Oriente: Coyoacán, Iztacalco, Iztapalapa; Poniente: Álvaro Obregón, Benito Juárez, Cuajimalpa, Magdalena Contreras, Miguel Hidalgo y Sur: Milpa Alta, Tláhuac, Tlalpan y Xochimilco.

* Trastornos mentales y del comportamiento, incluye trastorno bipolar, esquizofrenia, depresión y Alzheimer.

Enfermedades crónico-degenerativas, incluye diabetes mellitus, síndrome metabólico, disfunción eréctil, osteoporosis, esclerosis lateral amiotrófica, úlcera duodenal, ceguera, cataratas, glaucoma, cáncer de mama, cáncer de próstata, cáncer gástrico y cáncer de vejiga.

Enfermedades infecciosas/respiratorias, incluye neumonía, influenza, resfriado común, otitis media y virus papiloma $\mathrm{H}$

$\mathrm{RM}=$ Razón de momios; $\mathrm{SM}=$ Salario mínimo.

una prevalencia de consumo de alcohol en $34.7 \%$ y tabaco alguna vez en la vida en $46.3 \%$. ${ }^{6}$ Por otro lado, con base en la información de la Encuesta Nacional de Salud y Nutrición (ENSANUT-2006), se estimó la prevalencia del consumo de 100 cigarrillos en la vida en población de 60 años y más en hombres $(50.4 \%)$ y mujeres $(13.1 \%)$, así como el consumo diario de alcohol en hombres $(11.9 \%)$ y mujeres $(0.6 \%) .{ }^{5}$ Respecto a los problemas relacionados al consumo de alcohol, se halló una prevalencia del $2.8 \%$ en adultos mayores de 65 años con base en el uso del instrumento CAGE. ${ }^{6}$ En otras investigaciones se ha mostrado sólo una prevalencia de consumo riesgoso de alcohol en $9.6 \%{ }^{24}$ 
Investigaciones internacionales han identificado variaciones en las prevalencias de bebedores antes de los 65 años y de consumo riesgoso; el consumo en los siguientes países varió considerablemente: Cuba $(18.2 \% ; 7.4 \%)$, República Dominicana $(39.3 \% ; 30.1 \%)$, Perú $(8.2 \% ; 1.8 \%)$, Venezuela $(45.6 \% ; 4.5 \%)$, China $(11.0 \% ; 4.6 \%)$ y la India $(17.2 \% ; 0.8 \%) .{ }^{24}$ Las variaciones también fueron evidentes entre países con respecto al consumo de tabaco alguna vez en la vida para Cuba (44.8\%), República Dominicana (47.7\%), Perú (16.8\%), Venezuela (42.6\%), China (28.3\%) y la India (39.8\%). Metaanálisis realizados sobre 48 estudios mostraron que la prevalencia del consumo de tabaco en adultos mayores de 60 o más fue aún menor en $13.5 \%$. No obstante, la relación continúa siendo mayor en hombres $(22.5 \%)$ que en mujeres $(8.7 \%) .{ }^{25}$ Otras estimaciones realizadas en siete centros urbanos de América Latina con respecto al consumo actual de tabaco reportaron una prevalencia promedio de $16.3 \% .^{7}$ Sobre el consumo de drogas no se tiene registro a nivel nacional de prevalencias en población de adultos mayores. En Estados Unidos, el consumo de drogas mostró variaciones en el consumo de medicamentos sin prescripción entre comunidades de adultos mayores de 65 años (entre 31 y 96\%) ${ }^{20}$ y una muy baja prevalencia en el consumo de drogas ilícitas como la marihuana $(0.7 \%)$ y la cocaína $(0.04 \%)$ en los últimos 12 meses. En conjunto, los inhalantes, alucinógenos, metanfetaminas y la heroína representaron un consumo por debajo del $0.2 \%$. $^{21,26}$

El consumo de sustancias en adultos mayores de la Ciudad de México es menor cuando se compara con los resultados para la población de adultos (18 y 65 años) de la última Encuesta Nacional de Adicciones (ENA-2011) (Anexo 1). El consumo de alcohol alguna vez en la vida en la ENA en población adulta $(77.1 \%)^{2}$ fue $11.8 \%$ más alto en comparación con la estimación que se obtuvo en el presente estudio. Para el consumo de alcohol en los últimos doce meses fue mayor la prevalencia en $23.4 \%$ en adultos de la ENA (55.7\%) que en nuestro estudio, mientras que el abuso o dependencia al alcohol alguna vez en la vida $(8.6 \%)^{2}$ fue menor en la ENA en sólo $0.4 \%$ respecto a las estimaciones de este estudio. En cuanto al consumo de tabaco, el consumo alguna vez en la vida $(53.43 \%)$ y en los últimos doce meses $(23.6 \%)^{3}$ fue mayor, comparado con las estimaciones de este estudio (45.4 y $9.5 \%$, respectivamente). El consumo de drogas en los últimos 12 meses fue casi tres veces más prevalente en adultos $(2.8 \%)^{4}$ que en los adultos mayores según el presente estudio $(1.0 \%)$.

Con respecto a los factores asociados con el consumo de sustancias, se encuentra un consumo de alcohol más elevado en hombres (16.41\% hombres vs. $3.31 \%$ mujeres), tabaco (64.8\% hombres vs. $20.2 \%$ mujeres), ${ }^{27}$ y drogas ilícitas tanto en la bibliografía nacional como internacional. ${ }^{20,28,29}$ Un estudio realizó proyecciones de consumo de sustancias hasta el año 2020, y halló que en general es más probable que los adultos mayores varones presenten trastornos por abuso de sustancias, en particular en el rango de edad de los 50 a los 64 años. En poblaciones civiles no institucionalizadas, los adultos mayores en el rango de 50-59 años son quienes más tienden a usar drogas ilícitas o no médicas que aquéllos de mayor edad. La marihuana y la cocaína figuran entre las drogas ilícitas de mayor consumo, y los analgésicos opioides entre los medicamentos de prescripción médica mayormente usados por razones no médicas..$^{29}$ Otros factores relacionados con el abuso de sustancias en adultos mayores están asociados a ser del género femenino, presentar aislamiento social, historial de abuso de sustancias, historial de enfermedad mental, y/o exposición médica a drogas médicas de prescripción con abuso potencial. ${ }^{30}$

El uso de drogas médicas predomina en las mujeres, en especial en las mujeres adultas. ${ }^{20,31}$ Se piensa que en las mujeres los trastornos por uso y abuso de sustancias pueden estar relacionados con un mayor uso de servicios y acceso de las mujeres a psicofármacos de prescripción. En especial, a los analgésicos narcóticos de uso no médico y a los tranquilizantes. ${ }^{32}$ Las mujeres mayores de 65 años con deficiencia cognitiva, trastornos de pánico e ideaciones suicidas tienen mayores probabilidades de desarrollar dependencia a las benzodiazepinas. Investigaciones en los Estados Unidos han encontrado que las mujeres blancas con un nivel adquisitivo alto, discapacidad en las funciones físicas, historial de infartos o ataques al corazón, cuyo número de visitas a los centros de salud iba en incremento y presentaban obesidad eran mayormente asociadas al uso de analgésicos sin prescripción. Otros factores, como la mayor disponibilidad de recursos económicos que pueden ser destinados a la compra de alcohol y tabaco, son más controvertidos en mujeres, ya que hay escasas evidencias para esta población. Destaca, sin embargo, la comorbilidad entre el uso de sustancias ilícitas o fuera de prescripción y el consumo de alcohol. ${ }^{32}$

La investigación aquí presentada tiene limitaciones importantes. En primer lugar, está limitada a las personas de la tercera edad de sólo una entidad de la República. Esta investigación sólo consideró a participantes del programa de pensión alimentaria del Distrito Federal. Como este programa no tiene el $100 \%$ de cobertura, es posible que aquellas personas que se encuentran en los extremos socioeconómicos estén subrepresentadas en nuestro universo de investigación. En segundo lugar, es de considerar que aunque la muestra final resulta de tamaño adecuado, la prevalencia de consumo de algunas sustancias resultó infrecuente, especialmente aquélla de drogas ilícitas, lo cual impidió realizar análisis más detallados de consumo de estas sustancias en esta población. En tercer lugar, deben considerarse factores especiales debidos al segmento poblacional. Dado que nuestra investigación se basó en el autorreporte del uso de sustancias de los adultos mayores, y en esta población son comunes los factores relacionados a pérdidas cognoscitivas, las prevalencias aquí reportadas pueden verse afectadas y muy probablemente ser subestimadas. De hecho, como 
nuestra población bajo estudio presenta muchas de las enfermedades asociadas al proceso de envejecimiento, para evitar el cansancio en esta población nuestro cuestionario tuvo que limitarse en el número de variables relacionadas con el uso de sustancias. En cuarto lugar, es importante considerar la estructura del cuestionario. Aunque nuestro instrumento empleó definiciones de uso riesgoso de sustancias comunes en el área adecuadas para población general, es posible que estas definiciones usen puntos de corte poco adecuados para la población de la tercera edad y que estén subestimando el consumo riesgoso en este grupo.

Por último, es importante considerar también que esta investigación, al ser transversal y al ser realizada con población de tercera edad, privilegia a aquellas personas con sobrevida elevada, y da pie a que la posibilidad de una causalidad inversa sea un peligro latente. Por ello, se presentaron los resultados en forma conservadora, evitando en lo posible expresiones de causalidad al reportar nuestras asociaciones.

A pesar de las limitaciones que se mencionaron, las aportaciones de esta investigación son considerables. Éste es el primer estudio poblacional que se realiza en el país con población de tercera edad, con un domicilio fijo no institucionalizado, para conocer el consumo de sustancias psicoactivas. Se halló que el consumo de alcohol, incluso el consumo riesgoso, no fue infrecuente, el hábito tabáquico no ha desaparecido con el envejecimiento y el uso de drogas médicas puede ser un motivo de preocupación por su efecto en otras patologías. Esto revela la necesidad de establecer definiciones de uso riesgoso de sustancias para esta población y desarrollar programas de intervención adecuados para las características y necesidades de este grupo de edad.

\section{AGRADECIMIENTOS}

Los autores agradecen a la licenciada Rosa Icela Rodríguez Velázquez por el apoyo brindado (Directora General del IAAM-DF durante la realización del estudio) y al ingeniero Rubén Fuentes Rodríguez por el apoyo logístico territorial otorgado; a los médicos de la Coordinación de Geriatría por su participación en la capacitación del personal de campo y a todos/as los/las Coordinadores Regionales de las distintas delegaciones y a los educadores/as comunitarios por el trabajo en campo para el levantamiento de las entrevistas. Al equipo de trabajo de la Dirección Ejecutiva de Investigación y Evaluación del IAPA por el apoyo en la captura de los cuestionarios, muy especialmente a los maestros Enrique Aguilar Bustos y Andrea Gallegos Cari. Principalmente a todos los adultos mayores que tan generosamente abrieron sus puertas y compartieron con nosotros su información. Este trabajo fue financiado por el Instituto para la Atención y Prevención de Adicciones en la Ciudad de México. El reporte completo del estudio al que hace referencia, puede ser consultado vía web en la página: www.iapa.df.gob.mx. Si el lector desea conocer las preguntas del cuestionario de la encuesta dirija su petición al investigador responsable, en la correspondencia citada en el artículo.

\section{REFERENCIAS}

1. Medina-Mora Icaza ME, De la Parra A, Terroba G. The consumption of alcohol in the population of the Federal District. Salud Publica Mex 1980;22281-22288.

2. INPRFM, INSP, SSa. Encuesta Nacional de Adicciones 2011: Reporte de alcohol. México, DF: 2012.

3. INPRFM, INSP, SSa. Encuesta Nacional de Adicciones 2011: Reporte de Tabaco. México, DF: 2012.

4. INPRFM, INSP, SSa. Encuesta Nacional de Adicciones 2011: Reporte de Drogas. México, DF: INPRFM; 2012.

5. Shamah-Levy T, Cuevas-Nasu L, Mundo-Rosas V et al. Health and nutrition status of older adults in Mexico: results of national probabilistic survey. Salud Publica Mex 2008;50(5):383-389.

6. Aguilar-Navarro SG, Reyes-Guerrero J, Borges G. Cognitive impairment and alcohol and cigarette consumption in Mexican adults older than 65 years. Salud Publica Mex 2007;49s467-s474.

7. Kim S, De la Rosa M, Rice C, Delva J. Prevalence of smoking and drinking among older adults in seven urban cities in Latin America and the Caribbean. Subst Use Misuse 2007;42(9):1455-1475.

8. Lim SS, Vos T, Flaxman AD et al. A comparative risk assessment of burden of disease and injury attributable to 67 risk factors and risk factor clusters in 21 regions, 1990-2010: a systematic analysis for the Global Burden of Disease Study 2010. The Lancet 2013;380(9859):2224-2260.

9. INEGI, página web; 2010, consultado en http://www.inegi.org.mx/sistemas/olap/proyectos/bd/consulta.asp? $p=17118 \& c=27769 \& s=e s t \#$ el 1 de septiembre del 2013.

10. Portal ciudadano del Gobierno del Distrito Federal, página web; 2013, consultado en http://www.adultomayor.df.gob.mx/transparencia/padron.html el 14 de marzo del 2013.

11. CONAPO, página web; 2013, consultado en http://www.conapo.gob.

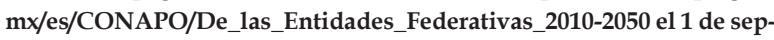
tiembre del 2013.

12. Portal ciudadano del Gobierno del Distrito Federal, página web; 2010, consultado en http://www.adultomayor.df.gob.mx/transparencia/padronjul2012.html el 14 de marzo del 2013.

13. Ham Chande R. Un siglo de cambios rumbo al envejecimiento. En: Ham Chande R (eds). El envejecimiento en México: el sigueinte reto de la transición demográfica. México: Porrua;2003.

14. CONAPO. Dinámica y prospectivas del envejecimiento sociodemográfico. En: CONAPO (eds). Diagnóstico sociodemográfico del envejecimiento en México. Serie de documentos técnicos. México, DF: 2011.

15. Wong R. Envejecimiento en áreas urbanas marginales de México: condiciones mixtas de privilegio y desventaja. En: Salgado de Snyder VN, Wong $R$ (eds). Envejecimiento, pobreza y salud en población urbana: un estudio en cuatro ciudades de México. Cuernavaca, Mor: INSP; 2006.

16. American Psychiatric Association. Diagnostic and Statistical Manual of Mental Disorder (DSM-IV-TR). Washington, DC: 2000.

17. Hosmer DW, Lemeshow S. Applied logistic regression. 2nd. New York, NY: Wiley \& Sons; 2000.

18. Solís LR, Vélez A, Berumen D. Prevalencia del consumo de bebidas alcohólicas y problemas asociados en personas de la tercera edad que acuden a los servicios de atención primaria de la Ciudad de México. Salud Mental 2000;23(3):38-45.

19. Contribuciones a las ciencias sociales, página web; 2010 , consultado en http://www.eumed.net/rev/cccss/07/ggvmsp.htm el 25 de abril del 2013.

20. Hanlon JT, Fillenbaum GG, Ruby CM, Gray $S$ et al. Epidemiology of over-the-counter drug use in community dwelling elderly. Drug Aging 2001;18(2):123-131.

21. Blazer DG, Wu LT. The epidemiology of substance use and disorders among middle age and elderly community adults: National Survey on Drug Use and Health (NSDUH). Am J Geriatr Psychiatry 2009;17(3):237-245. 
22. Wong R, Espinoza M, Palloni A. Adultos mayores mexicanos en contexto socioeconómico amplio: salud y envejecimiento. Salud Publica Mex 2007;49(4):436-447.

23. Wong R, Espinoza M, Palloni A. Adultos mayores mexicanos en contexto socioeconómico amplio: salud y envejecimiento. Salud Publica Mex 2007;49(436):447.

24. Ferri CP, West R, Moriyama TS et al. Tobacco use and dementia: evidence from the 1066 dementia population based serveys in Latin America, China and India. Int J Geriatr Psychiatry 2011;26(11):1177-1185.

25. Marinho V, Laks J, Coutunho ESF, Blay SL. Tobacco use among the elderly: a systematic review and meta-analysis. Cad Saude Publica 2010;26(12):2213-2233.

26. Blazer DG, Wu LT. The epidemiology of at-risk and binge drinking among middle-age elderly community adults national survey on drug use and health. Am J Psychiatry 2000;166(10):1162-1169.
27. Gallegos-Carrillo K, García-Peña C, Duran-Muñoz C, Reyes H et al. Autopercepción del estado de salud: una aproximación a los ancianos en México. Cad Saude Publica 2006;40(4):792-801.

28. Galvan EH, Caetano R. Alcohol use and related problems among ethnic minorities in the United States. Alcohol Res Health 2003;27(1):87-94.

29. Wu LT, Blazer DG. Illicit and nonmedical drug use among older adults: a review. J Aging Health 2011;23(3):481-504.

30. Culberson JW, Ziska M. Prescription drug misuse/abuse in the elderly. Geriatrics 2008;63(9):22-31.

31. Blow FC, Barry KL. Use and misuse of alcohol among older women. Alcohol Res Health 2002;4(308):315.

32. Simoni-Wastila L, Ritter G, Strickles G. Gender and other factors associated with the nonmedical use of abusable prescription drugs. Subst Use Misuse 2004;39(1):1-23.

Artículo sin conflicto de intereses

\begin{tabular}{|c|c|c|c|c|c|c|c|c|c|}
\hline \multicolumn{10}{|c|}{$\begin{array}{l}\text { ANEXO } 1 \\
\text { Prevalencia de consumo de sustancias y problemas asociados, alguna vez en la vida. } \\
\text { Cuadro comparativo entre la USAM-DF y la Encuesta Nacional de Adicciones (ENA) } 2011\end{array}$} \\
\hline & \multicolumn{4}{|c|}{ Alcohol } & \multicolumn{2}{|c|}{ Tabaco } & \multicolumn{3}{|c|}{ Drogas } \\
\hline$n$ & $\begin{array}{c}\text { Consumo } \\
\text { alguna vez } \\
\text { en la vida } \\
\% \\
\text { (IC) }\end{array}$ & $\begin{array}{c}\text { Consumo } \\
\text { en los } \\
\text { últimos } \\
12 \text { meses } \\
\% \\
\text { (IC) }\end{array}$ & $\begin{array}{c}\text { Consumo } \\
\text { riesgoso al } \\
\text { menos una } \\
\text { vez en la vida* } \\
\% \\
\text { (IC) }\end{array}$ & $\begin{array}{c}\text { Abuso o } \\
\text { dependencia } \\
\text { alguna vez } \\
\text { en la vida } \\
\% \\
\text { (IC) }\end{array}$ & $\begin{array}{c}\text { Consumo } \\
\text { alguna vez } \\
\text { en la vida } \\
\% \\
\text { (IC) }\end{array}$ & $\begin{array}{c}\text { Consumo } \\
\text { en los } \\
\text { últimos } \\
12 \text { meses } \\
\% \\
\text { (IC) }\end{array}$ & $\begin{array}{c}\text { Drogas } \\
\text { ilegales en } \\
\text { los últimos } \\
12 \text { meses } \\
\% \\
\text { (IC) }\end{array}$ & $\begin{array}{c}\text { Cualquiera } \\
\text { en los } \\
\text { últimos } \\
12 \text { meses } \\
\% \\
\text { (IC) }\end{array}$ & $\begin{array}{c}\text { Marihuana } \\
\text { en los } \\
\text { últimos } \\
12 \text { meses } \\
\% \\
\text { (IC) }\end{array}$ \\
\hline USAM-DF 2098 & $\begin{array}{c}65.3 \\
(63.2-67.3)\end{array}$ & $\begin{array}{c}32.3 \\
(30.3-34.3)\end{array}$ & $\begin{array}{c}27.1 \\
(25.2-29.1)\end{array}$ & $\begin{array}{c}9.0 \\
(7.8-10.3)\end{array}$ & $\begin{array}{c}45.4 \\
(43.2-47.5)\end{array}$ & $\begin{array}{c}9.5 \\
(8.3-10.8)\end{array}$ & $\begin{array}{c}0.2 \\
(0.1-0.5)\end{array}$ & $\begin{array}{c}1.0 \\
(0.7-1.6)\end{array}$ & $\begin{array}{c}0.1 \\
(0.0-0.3)\end{array}$ \\
\hline $\begin{array}{l}\text { ENA } \\
2011^{2,3,4}\end{array}$ & $\begin{array}{c}77.1 \\
(75.9-78.4)\end{array}$ & $\begin{array}{c}55.7 \\
(54.3-57.2)\end{array}$ & $\begin{array}{c}35 \\
(33.6-36.5)\end{array}$ & $\begin{array}{c}8.6 \\
(\mathrm{NIC})\end{array}$ & $\begin{array}{c}53.3 \\
(51.1-55.7)\end{array}$ & $\begin{array}{c}23.6 \\
(22.5-24.8)\end{array}$ & $\begin{array}{c}2.3 \\
(1.7-2.9)\end{array}$ & $\begin{array}{c}2.8 \\
(2.2-3.5)\end{array}$ & $\begin{array}{c}1.9 \\
(1.3-2.4)\end{array}$ \\
\hline \multicolumn{10}{|c|}{$\begin{array}{l}\text { Indicadores de USAM-DF representa la población entre 68-105 años del Distrito Federal. } \\
\text { Indicadores de ENA-201 } 1 \text { representa la población entre } 18-65 \text { años a nivel nacional. } \\
\text { * En la ENA se define como consumo alto. } \\
\text { NIC = No hay intervalo de confianza. } \\
\text { IC = Intervalos de confianza al } 95 \%\end{array}$} \\
\hline
\end{tabular}

